\title{
LAS PRIMERAS CENTRALES DE TELEFÓNICA EN GALICIA
}

\author{
Francisco Javier García Algarra
}

First Telefónica's Exchanges in Galicia

Boletín Académico. Revista de investigación y arquitectura contemporánea

Escuela Técnica Superior de Arquitectura. Universidade da Coruña

elSSN 2173-6723

www.boletinacademico.com

Número 2 (2012)

Páginas 01-08

Fecha de recepción 08.10.2011

Fecha de aceptación 17.01.2012

\section{Resumen}

Telefónica construyó tres centrales en Galicia entre 1928 y 1930 para alojar los equipos de la nueva red automática. Fueron diseñadas por José María de la Vega Samper, que tuvo una gran influencia en el desarrollo de este tipo de arquitectura en Espańa. Las centrales telefónicas son edificios industriales que esconden su naturaleza. Este artículo explica como siguieron el conjunto de principios de diseño que eran habituales en la arquitectura telefónica de Estados Unidos pero poco comunes en Europa.

\section{Abstract}

Telefónica built three telephone exchanges in Galicia between 1928 and 1930 to house the equipment of the new automatic network. They were designed by José María de la Vega Samper that has been very influential in the development of this kind of architecture in Spain. Central offices are industrial buildings that hide their nature. This paper explains how they followed the set of design principles that were common in the American telephone architecture but unusual in Europe.

\section{Palabras clave}

Central telefónica, arquitectura industrial, Telefónica, historicismo, influencia americana.

\section{Keywords}

Telephone Exchange, industrial architecture, Telefónica, historicism, American influence. 


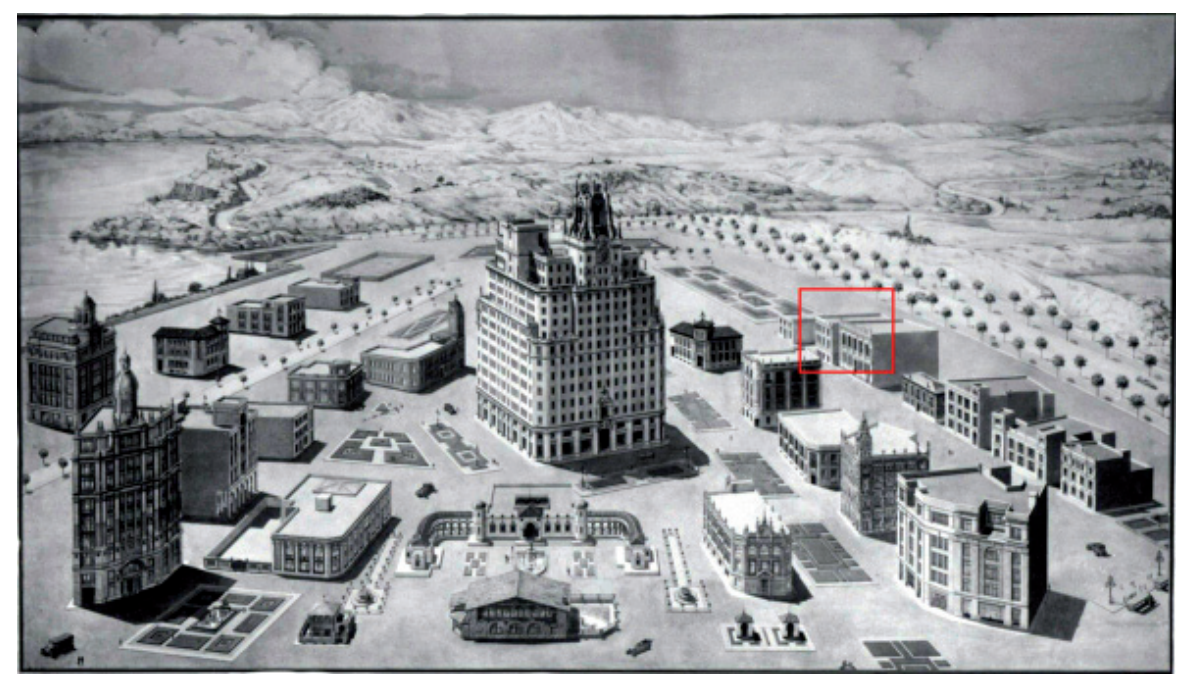

1 Ciudad Telefónica ideal, con las centrales de la CTNE en servicio en enero de 1930. Vigo aparece en el recuadro rojo.

\section{INTRODUCCIÓN}

El 25 de agosto de 1924, Alfonso XIII firmó el decreto que concedía a la Compañía Telefónica Nacional de España (CTNE), filial de la neoyorquina International Telephone and Telegraph Co. (ITT), el monopolio de explotación del servicio'. Como contrapartida, debía cumplir una serie de condiciones legales, económicas y técnicas recogidas en un contrato que se rubricó cuatro días después. La más importante era la construcción, en el plazo de cinco años, de una red de nueva planta, para la implantación de la telefonía automática en diecisiete ciudades (Fig. 01). En esa lista figuraban A Coruña y Vigo. Más tarde, la CTNE incluyó Santiago de Compostela en el plan.

La red automática necesita edificios de características constructivas especiales y con una distribución interior supeditada a las necesidades de los equipos. Es un tipo de arquitectura industrial muy particular, que utiliza la apariencia exterior para mimetizarse con el entorno urbano. La arquitectura telefónica tenía una larga tradición en Estados Unidos ${ }^{2}$. ITT implantó en su filial española una manera de trabajar similar a la que se seguía en su país de origen. Las centrales de Telefónica se parecen mucho más a las que se hacían en ese momento al otro lado del Atlántico que a las de Europa ${ }^{3}$.

Para cumplir los objetivos del contrato, Telefónica organizó un Departamento de Edificaciones en el que se gestaron la mayoría de los proyectos. Al frente estaba Ignacio de Cárdenas Pastor (1898-1979, t.1924), que acababa de terminar sus estudios de Arquitectura.
Aunque pueda parecer extraño, hay precedentes de situaciones similares en Estados Unidos.

Cárdenas se rodeó de un grupo de compañeros de su edad, la célebre generación de 1925. Algunos resultan desconocidos por haber circunscrito su actividad a Telefónica, como Paulino Justo Gayo Marín, Luis Clavero Margatí o Santiago García Claramunt. José María de la Vega Samper, que fue el autor principal de las tres centrales de Galicia, tuvo una obra prolífica también fuera de la Compañía. Resultan más familiares los nombres de Santiago Esteban de la Mora y Ramón Aníbal Álvarez, que formaron parte del GATEPAC. $\mathrm{Su}$ paso por Telefónica fue breve y las centrales que diseñaron (dos en Valencia, el primero, y la de Córdoba, el segundo) son bastante más convencionales que algunas de los del primer grupo ${ }^{4}$.

\section{SEMBLANZA BIOGRÁFICA}

José María de la Vega Samper (1900-1980, t.1924) nació en Toledo en el seno de una familia burguesa, sin precedentes de dedicación a la arquitectura. Ingresó en el Departamento de Edificaciones a las órdenes de Ignacio de Cárdenas, con el que había compartido aulas. Su firma aparece en algunos planos del proyecto del rascacielos de la Gran Vía madrileña de 1926. Antes de la Guerra Civil diseñó, solo o en colaboración, centrales en Madrid (Delicias), León, Pamplona, Salamanca, Valladolid, Gijón, Logroño, Vitoria y Lérida, además de las tres gallegas. Era capaz de manejar múltiples lenguajes, desde el historicismo de Salamanca y Santiago, a la experimentación racionalista en León. 

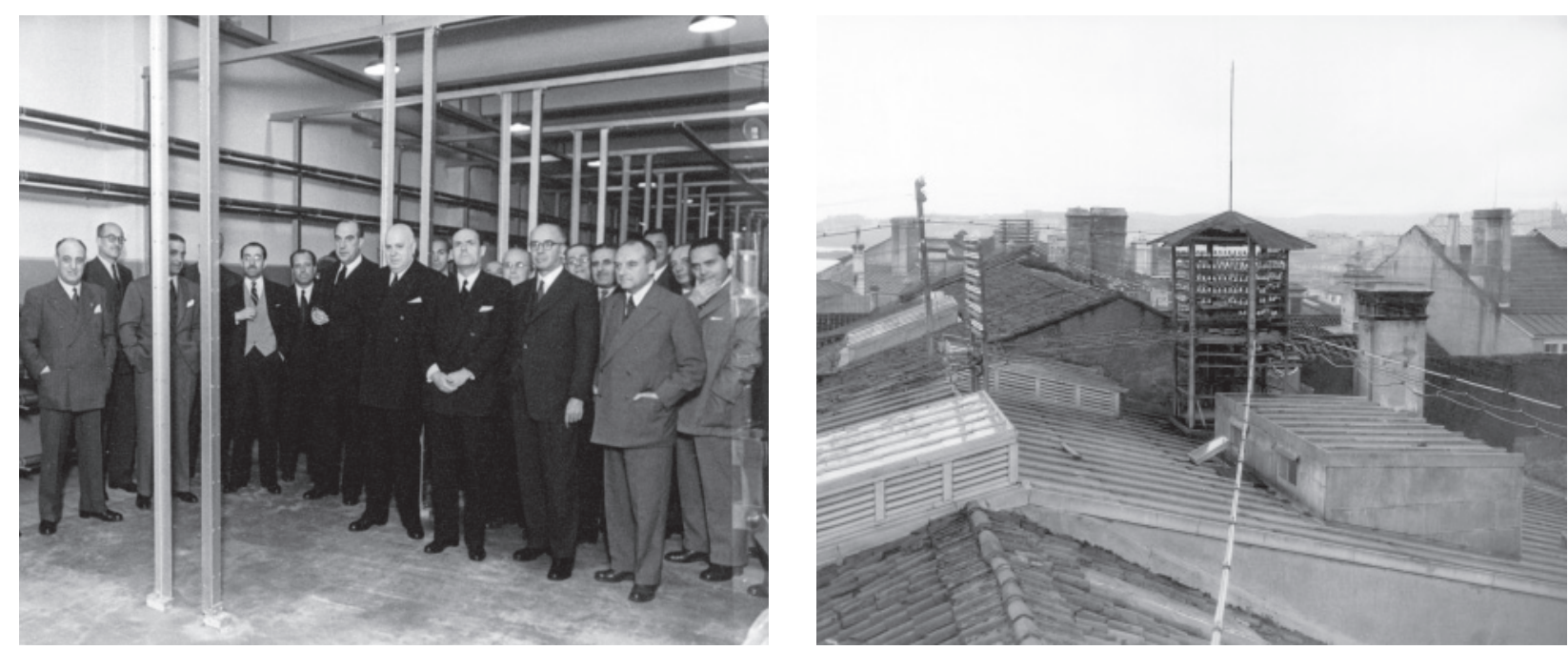

2 (Izda.) José María de la Vega Samper (primera fila, segundo por la derecha) en la inauguración de la central Norte de Madrid, 30 de abril de 1949.

3 (Dcha.) Viejo cableado aéreo en A Coruña, enero de 1928.

En julio de 1936 se refugió en la Embajada de Suecia, tras una breve detención. A mediados de 1938 estaba ya en la zona franquista trabajando como Arquitecto de Prisiones. Desde ese cargo participó con Luis de la Peña Hickman y Vicente Agustín Elguero en el diseño de la cárcel de Carabanchel (Madrid). A partir de 1939 sustituyó al frente del Departamento en la CTNE a Cárdenas, que se había exiliado, y sufrió depuración tanto por parte de la empresa como por parte del Colegio de Arquitectos ${ }^{6}$. Casi todas las centrales del periodo autárquico llevan su firma, y mantienen un estilo muy similar a las de antes del conflicto (Fig. 02). En paralelo, mantuvo abierto su propio estudio, que recibía múltiples encargos de la Iglesia y su entorno. Destaca el neoherreriano Colegio Mayor San Pablo, de Madrid (1943/51), realizado en colaboración con Luis García de la Rasilla Navarro-Reverter. La mayoría de los seminarios diocesanos de la posguerra salieron de su tablero de dibujo, destacando entre ellos el del Divino Maestro, en Ourense (1946/52).

A mediados de los años cincuenta abandonó el estilo imperial y recuperó el gusto por la modernidad de su juventud, en un proceso similar al de Luis Gutiérrez Soto. El Colegio Español de San José, en Roma $(1956 / 65)$ o la iglesia parroquial de los Sacramentinos en Madrid (1955/66), dan testimonio de esta transformación que se produjo también en sus diseños para Telefónica, como puede apreciarse en la central de Las Ventas en Madrid. Su actividad en este campo se redujo al comenzar los años sesenta. Siguió colaborando con la empresa hasta 1972, cuando firmó el proyecto de la central Peñuelas en Madrid.

\section{PRINCIPIOS DE DISEÑO}

En 1945, José María de la Vega Samper pronunció una conferencia ante los jefes técnicos de la Compañía; una conferencia valiosa, por tener la frescura de un documento interno ${ }^{7}$. Contiene una síntesis de la doctrina que guió al Departamento durante décadas, y una breve revisión crítica de los edificios construidos hasta entonces.

El punto de partida del proyecto siempre lo proporcionaban los ingenieros responsables del equipo automático, que indicaban al arquitecto la disposición óptima de los bastidores.

\footnotetext{
Si el edificio ha de ser central automática habrá de ser la Sección de Equipo del Departamento de Ingeniería la que dé las primeras referencias, ya que la instalación del equipo será la más importante en cuanto a extensión, coste y servicios. Será conveniente por tanto, que sobre un plano del solar determine la referida Sección de Equipos la mejor disposición para los bastidores, los cuales han de estar en un mismo local, siempre que la planta tenga la superficie suficiente para ello. ${ }^{8}$
}

Todas las salas de equipos son iguales, grandes recintos hipóstilos exentos con un módulo rectangular de 17x24 pies americanos. A partir de la distribución de la planta, se determinaba la mejor forma de cumplir con el resto de los requisitos.

Para ello ha de tenerse la información necesaria [de los departamentos de Tráfico, Comercial y Conservación] pero corrientemente, como se conocen, por la práctica de los edificios ya construidos de una manera bastante aproximada aquellas necesidades, ganaremos tiempo proponiendo una solución ya estudiada. ${ }^{9}$ 

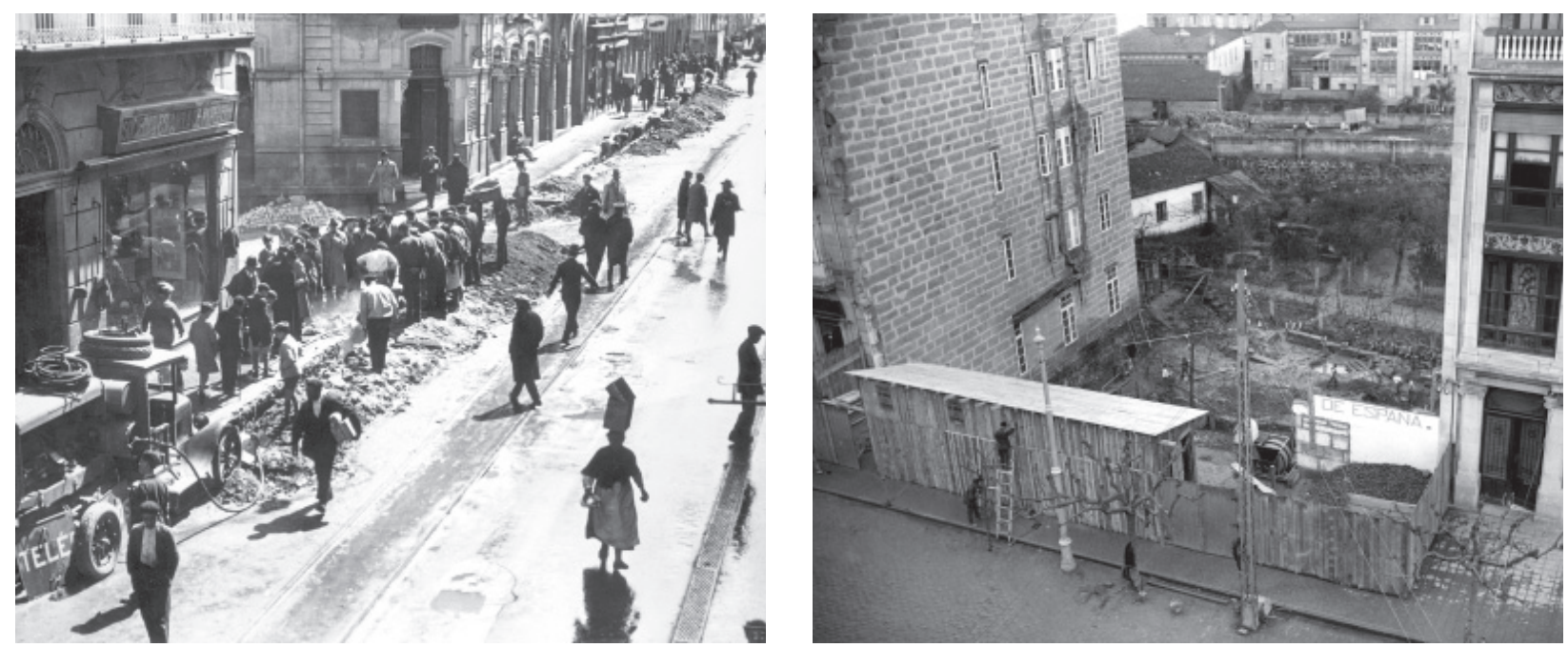

4 (Izda.) Excavación para el nuevo cableado subterráneo en Vigo, febrero de 1928.

5 (Dcha.) Comienzo de los trabajos de cimentación de la central telefónica de Vigo, febrero de 1928.

Este pasaje da una pista sobre una de las causas de la gran productividad alcanzada por el Departamento: la repetición de fórmulas de eficacia contrastada.

Resuelta la distribución general, el proyecto quedaba ya en manos del arquitecto, que sólo entonces empezaba a tener en cuenta la faceta estética.

Realizado el primer estudio de distribución ha de comenzar el Arquitecto a diseńar los alzados procurando armonizar la distribución interior, siendo este trabajo personalísimo suyo. [...] En cuanto a lo que el aspecto estético concierne, el Arquitecto ha de tener presente dos cosas fundamentales: primero, el edificio ha de tener carácter de Central telefónica y, segunda, ha de responder al ambiente arquitectónico de la región o lugar en que esté enclavado. ${ }^{10}$

A continuación se procedía a negociar con el ayuntamiento de la localidad.

A veces se consigue lo que se pretende, esto es, que el Ayuntamiento dé la licencia de construcción con lo que la Compañía necesita. Otras veces es necesario modificar las fachadas y, o bien construir en primera crujía algún aditamento con el menor fondo posible, como ocurre en el edificio de Huelva, o construir una especie de pérgola como se hizo en Alicante, o un torreón en una de las esquinas de lo que hay ejemplos en Salamanca, Gijón, etc. ${ }^{11}$

Estas torrecillas postizas terminaron convirtiéndose en una de las características de la arquitectura de la CTNE.

La actividad del Departamento fue muy intensa desde su puesta en marcha en 1926 hasta la proclamación de la República. En 1928 entraron en servicio dieciséis centrales automáticas instaladas en edificios de nueva construcción, y hasta 1932 se completaron cuarenta y dos, una cifra sobresaliente.

\section{LAS CENTRALES GALLEGAS}

Las centrales gallegas se proyectaron entre 1927 y 1928 y se construyeron de manera casi simultánea. Vigo se completó en 1929 y A Coruña y Santiago en 1930. José María de la Vega Samper fue el autor de los tres proyectos, con la colaboración en el segundo del arquitecto cubano Santiago García Claramunt. En todos ellos se empleó una estructura de hormigón armado, con forjados capaces de soportar $850 \mathrm{~kg} / \mathrm{m}^{2}$ en las salas de equipos. Era la práctica habitual de la CTNE, heredada de las normas americanas.

Los edificios son solo un elemento más de la red. El contrato de la CTNE con el Estado estipulaba que en el centro de las ciudades el cableado debía soterrarse, como se hacía en América del Norte. En España, hasta entonces, los cables telefónicos urbanos eran aéreos y se apoyaban en caballetes de madera o metal, instalados en tejados y azoteas (Fig. 03). La excavación de zanjas despertó una gran curiosidad en el público, como puede observarse en las fotografías de los trabajos en la vía pública (Fig. 04).

La central de Vigo (Fig. 05-06), que vivía entonces una época de gran desarrollo, se levanta en la calle Urzáiz. Edificios como La Peineta (1926) y el Garaje Americano (1929) de Jenaro de la Fuente Álvarez (1891-1963, t.1921), o el Círculo Mercantil e Indus- 

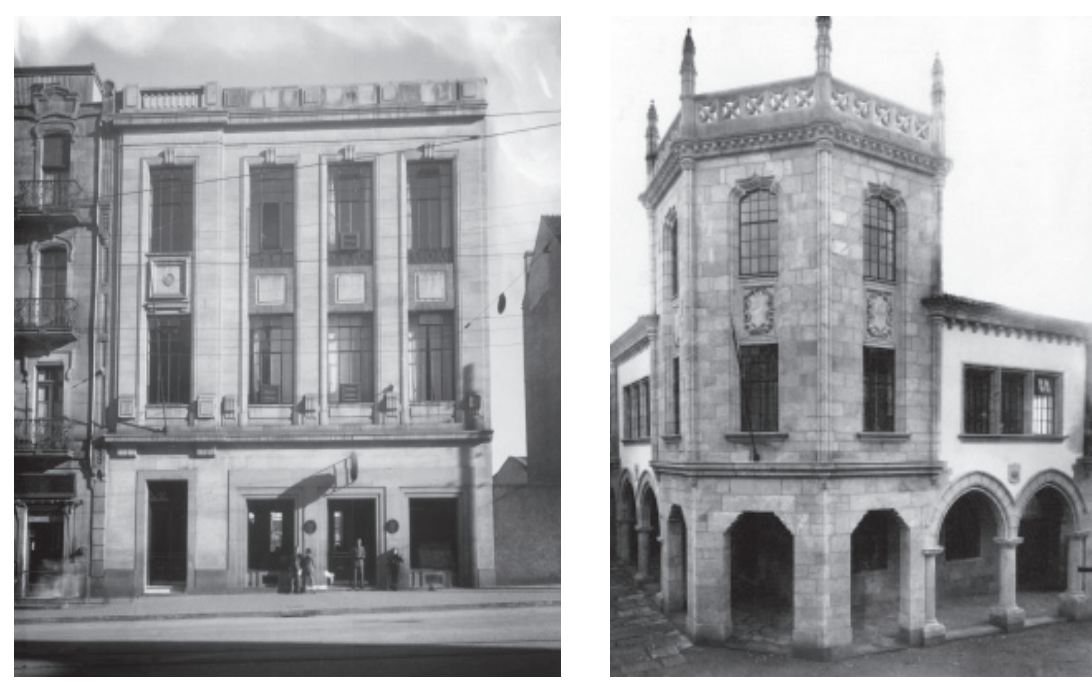

6 (Dcha.) Central telefónica de Vigo en la calle Urzáiz (1928/29). 7 (Izda.) Central telefónica de Santiago de Compostela (1928/30)

trial (1927) de Manuel Gómez Román (1876-1964, t.1917), hablan del gusto vigués por la arquitectura comercial de influencia anglosajona.

En este entorno, De la Vega encontró un ambiente propicio para su habilidad con el lenguaje a la americana. La distribución interna es la común en las centrales de tamańo medio: planta baja abierta al público con locutorio y oficina comercial, y pisos primero y segundo dedicados a equipos y servicios internos. La fachada principal se abre a Urzáiz, y presenta tres zonas diferenciadas. En la inferior se encuentra la puerta de acceso, entre dos escaparates. El cuerpo intermedio, separado del primero por una marcada línea de imposta, se organiza en cuatro calles en las que se abren amplios ventanales metálicos recercados en piedra. La de la izquierda, que sobresale ligeramente y se sitúa sobre la puerta, corresponde a la caja de escaleras. El antepecho presenta un adorno de cantería más elaborado que los del resto de la fachada, muy esquemáticos. El conjunto se corona con cornisa y balaustrada. Este remate resultaba conveniente porque, de hecho, la estructura se calculaba para soportar las previsibles ampliaciones en altura. Llegado el momento, era sencillo añadir plantas sin causar un gran prejuicio a la estética. La central de Vigo (llamada Calvario en el nomenclátor de Telefónica) tiene en la actualidad dos plantas más que en 1930.

Algunos de sus elementos característicos, como el cerramiento completo en granito, las puertas o la disposición de los ventanales, se repitieron en obras posteriores del autor.
La Revista Telefónica Española dedicó una extensa noticia a la inauguración de esta central, en la que no se menciona al arquitecto y en la que se incluyen varios pasajes de la crónica que publicó El Correo Gallego ${ }^{12}$. El periodista transmitía algunos detalles curiosos sobre el funcionamiento diario del centro, como el uso de un tubo neumático para la conducción de telefonemas (telegramas dictados por teléfono). Del edificio, sólo le llamó la atención la azotea, «desde la cual se domina una maravillosa perspectiva de nuestra bahía» ${ }^{13}$.

En Santiago de Compostela concurrían circunstancias especiales (Fig. 07). Sobre el solar cedido por el ayuntamiento en la calle del Franco, se había levantado la Casa Grande del Cabildo, una construcción del siglo $\mathrm{XV}$. La central debía incorporar los restos que quedaban de la portada y construirse en un estilo compatible con el del caserío del casco histórico compostelano (Fig. 08). Era un encargo apropiado para José María de la Vega, que dominaba como nadie en el Departamento los lenguajes históricos. En Salamanca (1927/29) ya había integrado los restos del Palacio de Solís en un edificio neoplateresco inspirado en el Palacio de Monterrey. Con motivo de la inauguración escribió:

El problema de proyectar un edificio en la Ciudad de Compostela tenía dos aspectos importantes: hacer un edificio que respondiese a las necesidades interiores del objeto para el que se levantaba y responder a las necesidades que pudiéramos decir externas, impuestas por el ambiente antiguo de Santiago.

[...] El hecho de que el Ayuntamiento manifestase su deseo de que en el nuevo edificio tuviera lugar adecuado la preciosa portada de estilo gótico que existía en el que hubo de derribarse, resolvía la cuestión de la elección de estilo. Pero por otro lado 

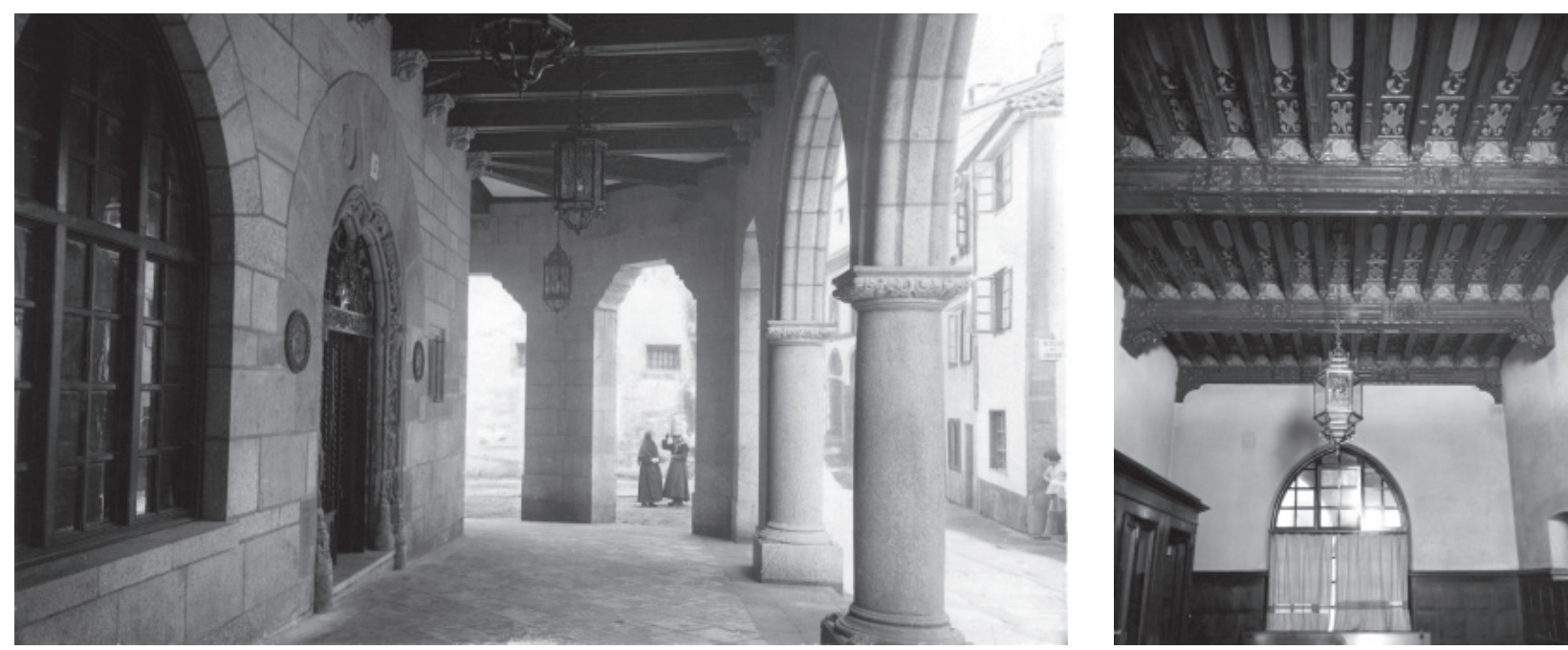

8 (Dcha.) Soportales en la calle del Franco. Puede verse la portada gótica de la Casa Grande integrada en el edificio, 1930. 9 (Izda.) Interior de la central telefónica de Santiago, con las viguetas de hormigón decoradas para simular madera antigua, 1930.

el predominante en las construcciones típicas de Santiago es el barroco y, por tanto, hubo de mezclarse de la manera más discreta posible algo de barroco en el conjunto gótico. ${ }^{14}$

La descripción pormenorizada de los detalles arquitectónicos en una noticia de inauguración es inusual en la Revista Telefónica Española, que se extendía en la transcripción de los discursos protocolarios y prestaba alguna atención al equipamiento, pero muy poca al continente.

Siendo el pórtico un elemento típico de las construcciones santiaguesas, se proyectó un edificio a base de dicho elemento, al cual se ha concedido excepcional importancia. El frente del mismo, que forma parte de la fachada, está formado por una arquería de columnas monolíticas de granito del país.

[...] El techo se ha decorado a base de viguetas de la estructura de hormigón, ornamentadas con detalles góticos simulando vigas de nogal, las cuales descansan sobre canecillos de piedra granítica con historiadas y originales tallas. ${ }^{15}$

El coste económico de esta efusión decorativa no puede explicarse más que por la voluntad de ostentación. Resulta anacrónico — pero no por ello hay que dejar de reconocer que creativo- el disfraz del lacunario de hormigón como artesonado de maderas nobles (Fig. 09).

[Todos los detalles de la fachada] han sido inspirados en antiguas construcciones de Santiago, lo mismo molduras que antepechos, pináculos, canecillos, escudos, etc. ${ }^{16}$

En 2006, Telefónica vendió a Correos este edificio, que ha sido reformado con gran acierto por Enrique Solana de Quesada, Galo Zayas Carvajal e Ismael Ameneiros Rodríguez y reinaugurado en $2010^{17}$.
La central de A Coruña en la calle de San Andrés, tiene una composición parecida a la de Santiago, con dos plantas y una torre en el chaflán rematada con balaustrada y pináculos (Fig. 10).

Este edificio, si se prescinde de dicha torre, hay que clasificarlo dentro de la línea mayoritaria de construcciones funcionales de la CTNE. La puerta principal, la decoración de la planta baja, los ventanales de la primera y los huecos acristalados con antepechos metálicos y remate en arco de medio punto se repiten en otras centrales. Es una obra de repertorio del Departamento de Edificaciones, un testimonio de su eficacia.

Sigue sirviendo al propósito para el que se construyó y ha experimentado dos ampliaciones. En la primera, se recrecieron las alas una planta, hasta enrasarlas con la torre. En la segunda, se añadió una nueva planta sobre el conjunto, con una torrecilla decorativa en la fachada de la calle de San Andrés.

\section{CONCLUSIONES}

Las tres centrales gallegas se proyectaron en el marco de la creación de una red telefónica automática en las principales ciudades españolas durante los años finales de la dictadura de Miguel Primo de Rivera. Son construcciones de propósito industrial con una organización interna impuesta por los equipos. Difieren en su aspecto exterior, que se adecuaba al del entorno para no provocar rechazo. La de Santiago es un ejercicio de historicismo, que mezcla motivos góticos y barrocos propios de la ciudad. En Vigo y A Coruña, por el con- 
trario, se eligió un ropaje más moderno y apropiado a su situación en dos arterias comerciales de primer orden. Son uno de los conjuntos más coherentes en la arquitectura telefónica anterior a la Guerra Civil, por ser obra del mismo arquitecto, José María de la
Vega Samper. En los años cincuenta, De la Vega fue responsable del diseño de las centrales automáticas de Ourense, Pontevedra, Lugo y Ferrol. Todas ellas reproducen esquemas muy parecidos a la de Vigo, que sirvió también de modelo a otras fuera de Galicia.

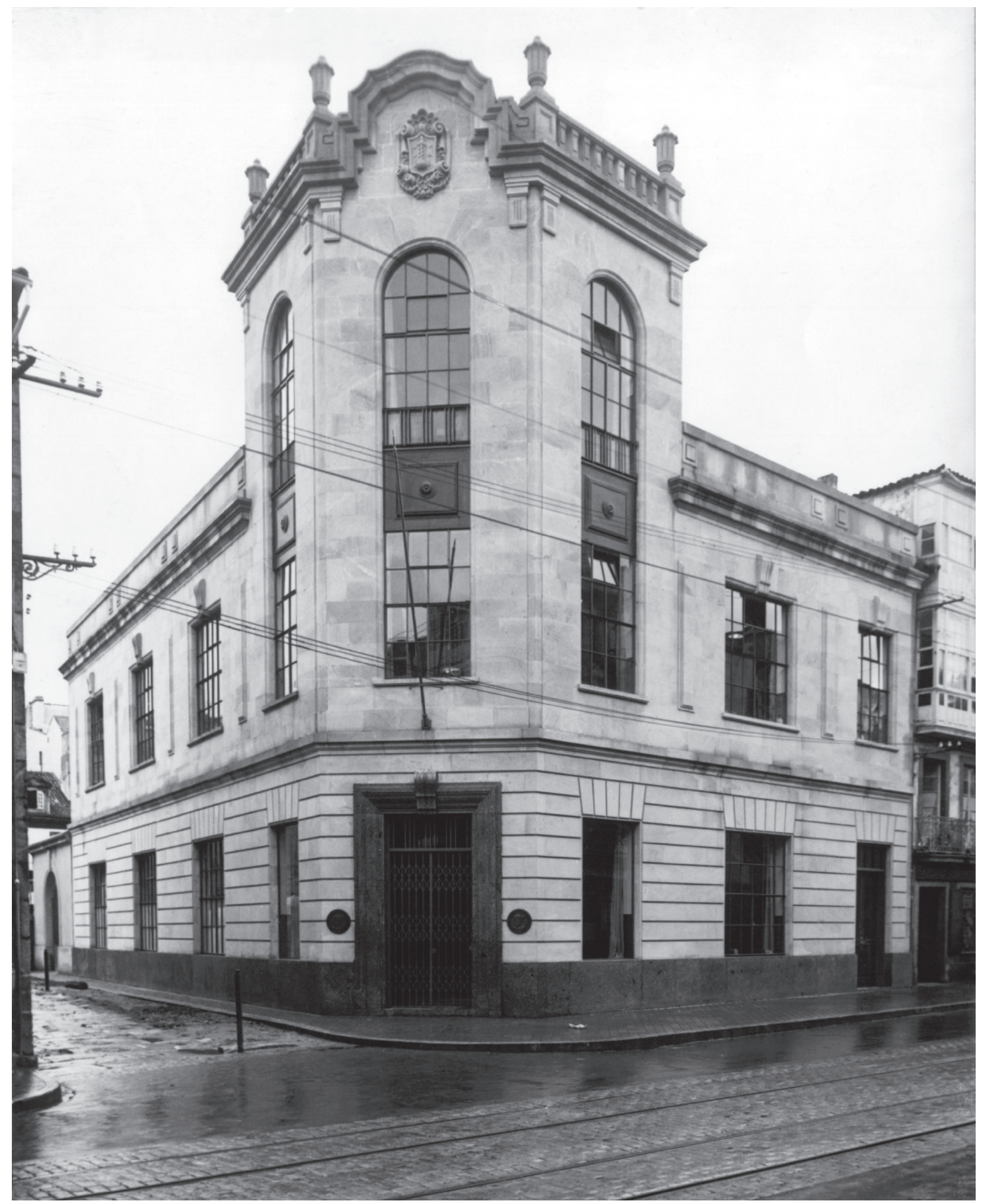

10 Central telefónica de A Coruña en la calle de San Andrés (1928/30). 


\section{Notas}

1. Cf. Antonio Pérez Yuste, «La creación de la Compañía Telefónica Nacional de España en la Dictadura de Primo de Rivera», Cuadernos de Historia Contemporánea 29 (2007): 95-117.

2. Esta expresión es la que empleaba el Departamento de Edificaciones de la CTNE para referirse a su trabajo.

3. Cf. Javier García Algarra, «Centrales telefónicas norteamericanas en los años 20», Espacio, Tiempo y Forma 16 (2003): $199-221$.

4. Algunos de los arquitectos del Departamento de Edificaciones de la CTNE participaron activamente en el movimiento para la colegiación profesional obligatoria (cf. COAM, Servicio Histórico, nota sobre el $75^{\circ}$ aniversario de la creación de los Colegios. Madrid, 27 de diciembre de 2004 ).

5. Se conserva una carta de julio de 1938 de Pedro Muguruza Otańo en la que informa de un proyecto de Vega Samper para construir un penal para mil reclusos en el Hospital de Santa Cruz de Toledo (cf. José Álvarez Lopera, «Realidad y propaganda: El patrimonio artístico de Toledo durante la Guerra Civil», Cuadernos de Arte e Iconografía 6 (1990): 91-108).

6. Cf. COAM, Junta de Gobierno. «Acuerdo adoptado en sesión 2003.J/24 celebrada el día 7 de julio por el que se deroga el proceso de depuración político-social de arquitectos iniciado en 1939».

7. Cf. José María de la Vega Samper: «Diseño y distribución de edificios telefónicos y su construcción». Conferencia n. 18 de la II Reunión de Jefes Técnicos, CTNE, julio de 1945. Archivo de la Fundación Telefónica.

8. Idem, 6 .

9. Loc. cit.

10. Idem, 8.

11. Idem, 8-9.

12. «El servicio automático en Vigo», Revista Telefónica Española 10 (1929): 5-17. Noticias de la inauguración también se pueden encontrar en La Vanguardia, 15 de junio de 1929, 30.

13. «El servicio automático en Vigo", op. cit., 15.

14. «El automático en Santiago de Compostela», Revista Telefónica Española 1 (1931): 19-29.

15. Idem, 26-27.

16. Idem, 28 .

17. Cf. Jaime Alarcón, Antonio Blanco: «Reforma integral del edificio principal de Correos en Santiago de Compostela». Tecno [OHL, revista interna de formación e innovación] 75 (2010): 28-43. Se indica que «[El edificio de Telefónica] está catalogado dentro del Nivel 4 del Catálogo de Plan Estatal de Protección y Rehabilitación de la Ciudad Histórica».

\section{Procedencia de las ilustraciones}

Todas las fotografías pertenecen al Archivo Histórico Fotográfico de Telefónica, que ha autorizado su uso para este artículo.

Fig. 01. Negativo R-05588. Apareció en la Revista Telefónica Española en 1930.

Fig. 02. Negativo R-01008. Autor: desconocido.

Fig. 03. Negativo 744. Autor: Marín.

Fig. 04. Negativo R-02311. Autor: Marín.

Fig. 05. Negativo R-00725. Autor: Marín.

Fig. 06. Negativo R-00721. Autor: desconocido.

Fig. 07. Negativo R-00743. Autor: Arturo.

Fig. 08. Negativo R-05523. Autor: Arturo.

Fig. 09. Negativo R-00748. Autor: Arturo.

Fig. 10. Negativo R-00746. Autor: desconocido.

\section{Sobre el autor}

Francisco Javier García Algarra (Madrid, 1965). Ingeniero de Telecomunicación (UPM, 1990), Licenciado en Geografía e Historia (UNED, 2001) y Máster en Historia del Arte (UNED, 2001). Desde 1991 desarrolla su actividad profesional en Telefónica Investigación y Desarrollo. Acaba de registrar su tesis doctoral sobre el patrimonio arquitectónico de Telefónica, realizada en el Departamento de Historia del Arte de la UNED.

algarra@tid.es 